\title{
Carnets
}

Revue électronique d'études françaises de l'APEF

Première Série - 4 Numéro Spécial | 2012

Invasions \& Évasions

\section{Invasions Françaises ou les modalités de présence du théâtre français au Portugal}

\section{Ana Clara Santos}

\section{OpenEdition}

Journals

Édition électronique

URL : http://journals.openedition.org/carnets/7682

DOI : 10.4000/carnets.7682

ISSN : 1646-7698

Éditeur

APEF

Édition imprimée

Date de publication : 1 juin 2012

Pagination : 207-218

Référence électronique

Ana Clara Santos, «Invasions Françaises ou les modalités de présence du théâtre français au

Portugal », Carnets [En ligne], Première Série - 4 Numéro Spécial | 2012, mis en ligne le 23 juin 2018 , consulté le 01 mai 2019. URL : http://journals.openedition.org/carnets/7682 ; DOI : 10.4000/ carnets.7682

\section{(c) (i) (8)}

Carnets est mis à disposition selon les termes de la licence Creative Commons - Atribution - Pas d'utilisation commerciale 4.0 International. 


\title{
INVASIONS FRANÇAISES OU LES MODALITES DE PRESENCE DU THEATRE FRANÇAIS AU PORTUGAL
}

\author{
ANA CLARA SANTOS \\ Université d'Algarve \\ $\mathrm{CET}^{1} /$ Université de Lisbonne \\ avsantos@ualg.pt
}

\begin{abstract}
Résumé
S'il faut attendre une dizaine d'années après le départ des troupes napoléoniennes pour assister à une certaine effervescence dans le domaine théâtral, grâce à la collaboration entre impresarios et salles de spectacles à Paris et à Lisbonne, il faut bien avouer que cette influence, matérialisée par la dispute entre quelques compagnies théâtrales et le renouvellement persistant du répertoire des salles de spectacle dans la capitale portugaise, constitue un facteur essentiel vers un repositionnement du regard qu'il faut désormais projeter sur l'évolution de l'histoire culturelle au Portugal à cette époque. En effet, bien que le répertoire parisien soit à l'origine de plus de $80 \%$ des représentations sur la scène à Lisbonne, c'est surtout l'importation d'une pratique théâtrale et d'un certain savoir-faire qui aura un impact mesurable sur le monde théâtral portugais à l'aube du Romantisme.
\end{abstract}

\begin{abstract}
Ten years after the departure of Napoleon's troops, a certain enthusiasm in this area began to be noticed as a result of the collaboration between managers and theatre halls in Paris and Lisbon. This influence was admittedly marked by the dispute between several theatre companies and the constant renewal of the repertoire in the Portuguese capital's theatres, and it constitutes a key factor in the new outlook which needs to be addressed in terms of the evolution of cultural history in Portugal at that time. Although the repertoire in the Parisian scene accounted for more than $80 \%$ of performances on Lisbon stages, it was above all the import of theatrical practices and some know-how that were to have a measurable impact on the Portuguese theatrical world on the dawn of Romanticism.
\end{abstract}

Mots-clés: compagnies théâtrales, répertoire, traduction, spectacle.

Keywords: theatre companies, repertoire, translation, performance.

\footnotetext{
${ }^{1}$ Cette recherche s'insère dans un projet de recherche sur la réception de la dramaturgie et du théâtre français au Portugal que l'on développe au sein du Centre d'Études de Théâtre (CET) de l'université de Lisbonne.
} 
Le contact maintenu entre la culture portugaise et la culture française est, comme chacun sait, très ancien. Dans le champ culturel artistique, ces liens se renforcent au cours du XVIIle siècle. À l'époque de l'llluminisme, les livres et les ouvrages des encyclopédistes français circulent au sein de la société cultivée portugaise malgré la forte censure exercée par les structures de l'État comme la Intendência Geral da Polícia do Reino, le Tribunal do Santo Ofício et, bien sûr, la Mesa Real Censória. La langue française véhiculait, comme partout en Europe, les nouveaux idéaux d'une idéologie susceptible de rompre avec la tradition de l'Ancien Régime. De nouvelles modes françaises s'instaurent parmi lesquelles l'usage de la cocarde tricolore qui reste, quand même, l'une des plus symboliques. Nombreux sont les témoignages de ce phénomène d'acculturation qui durent jusqu'à l'entrée des troupes françaises napoléoniennes sur le territoire national. Carl Israel Ruders rend compte de ce phénomène dans son récit de voyage au Portugal et nous dit justement qu'en 1802, à partir de l'arrivée de l'ambassadeur Lannes, on voyait à Lisbonne de nombreuses personnes qui portaient la cocarde tricolore. Certains documents officiels rehaussent aussi l'exemple militaire du comte d'Assumar (D. Pedro de Almeida Portugal, 3ème marquis d'Alorna e 5ème comte d'Assumar) qui renforce l'usage de ce symbole dans les campagnes de son régiment à l'époque ${ }^{2}$.

\begin{abstract}
Portugal conta agora também, entre os principais enviados estrangeiros, um ministro de França. [...] O ministro francês é de grande estatura e bem conformado. Mostra-se com frequência trajando o esplêndido uniforme de general de cavalaria. Depois da sua chegada vêem-se por aqui muitas pessoas de cocar tricolor. Até o duque de Luxemburgo, sogro do duque de Cadaval, traz essa insígnia. Apesar disso, não pude conter o riso, quando uma destas manhãs vi o meu cabeleireiro francês, recentemente republicanizado, apresentar-se em minha casa com esse distintivo no chapéu, todo cheio de nódoas de pós e pomadas (Ruders, 2002: 267-268).
\end{abstract}

Or si on commence par évoquer ces questions liées à des phénomènes sociaux et militaires c'est qu'effectivement, à l'aube du XIXe siècle, on ne peut pas vouloir cerner la question artistique et culturelle sans aborder ces aspects qui ont marqué partout la vie en Europe. Comme d'autres pays européens, le Portugal subit les conséquences de l'élargissement de l'empire napoléonien et cette réalité politique et militaire n'est pas sans avoir des répercussions sur le plan culturel. Le pays a été occupé, comme on le sait, à trois reprises par les troupes de Napoléon. La 1ère campagne est commandée par le général

\footnotetext{
2 “(...) e nas guarnições das espadas o conde de Assumar mandou fundir para os oficiais do seu regimento (...) um cocard a que chama o povo o Barrete da Liberdade" (IAN/TT, 1794: 212).
} 
Junot qui arrive au Portugal le 19 novembre 1807, dix jours avant le départ de la cour portugaise en direction de sa colonie outre- atlantique, le Brésil. Cette stratégie portugaise qui, au départ, est instaurée pour préserver la souveraineté nationale, sera aussi, comme on verra plus loin, l'occasion d'une dynamisation culturelle par le biais de la circulation d'artistes de la métropole vers la colonie. Le rôle des anglais, commandés par Sir Arthur Wellesley, le futur Duc de Wellington, qui débarquent alors dans le pays et battent les armées de Napoléon à Roliça et à Vimeiro, au cours du mois d'août, est alors décisif pour la signature de la Convention de Sintra en vue du départ des troupes napoléoniennes en France. Ceci n'a pas été suffisant pour déjouer les plans de l'empereur français et seule la défaite de la deuxième campagne, commandée par le maréchal Soult, entre mars et mai 1809, le pousse à entreprendre une dernière tentative afin de soumettre le Portugal. La troisième campagne, commandée par le maréchal Masséna à laquelle appartiennent le maréchal Ney et le général Junot (août 1810-mars 1811), aura été la plus dévastatrice plongeant le pays dans la violence et la misère. Les conditions étaient alors réunies pour que s'instaure un système anarchique et s'amorce une lutte fratricide entre les anti-français et la poursuite des afrancesados. Dans ces conditions, beaucoup d'intellectuels sont forcés à l'exil, notamment vers le Brésil. Certains de ces artistes avaient été invités par le roi à traverser l'Atlantique afin d'édifier la cour artistique à Rio de Janeiro. Ce fut le cas du compositeur Marcos Portugal qui était maître de musique au théâtre S. Carlos depuis 1800. II arriva au Brésil le 11 juin 1811 et, nommé maître de musique du Roi, il est vite devenu le symbole de la mise en scène musicale du pouvoir Royal, responsable de sa splendeur et de sa grandeur dans le Théâtre et dans toutes les manifestations festives ou religieuses. De 1811 à 1830, date de sa mort, il resta une figure de référence dans le panorama musical luso-brésilien.

L'une des premières mesures royales fut l'édification d'un théâtre national, celui de $S$. João à Rio de Janeiro. Il fut inauguré à l'occasion de l'anniversaire du prince D. Pedro, le 12 octobre 1813, à la suite du décret daté de 28 mai 1810 qui promulgue sa création :

Fazendo-se absolutamente necessário nesta Capital que se erija um teatro decente, e proporcionando à população e ao maior grau de elevação e grandeza em que se acha pela minha residência nela, e pela concorrência de estrangeiros e outras pessoas que vêm das extensas Províncias de todos os meus Estados, fui servido encarregar o doutor Paulo Fernandes Viana, do meu Conselho e Intendente de Polícia, do cuidado e diligência de promover todos os meios para ele se erigir (apud Martins, 1978: 161).

À cette époque, se développent aussi les autres arts puisqu'en 1816, arrive à Rio, une mission artistique française dirigée par Lebreton, de l'Institut de France. Cette mission 
intègre quelques artistes de grande renommée: des sculpteurs, des architectes, des graveurs et des peintres, notamment Nicolas et Auguste Tauney, Montigny, Pradier et Debret. II faut dire que cette mission avait été orchestrée par l'ambassadeur à Paris, le marquis de Marialva, et avait pour objectif la fondation de la «Escola Real das Ciências, Artes e Ofícios » qui fut bientôt édifiée.

Le théâtre pris, d'un côté, dans son acception la plus large qui fut celle du XIXe siècle, lieu de divertissement et de sociabilité et, de l'autre, dans ses rapports interculturels institués avec les pays voisins, nous permettra de mesurer d'emblée l'apport du siècle de l'Empire à la culture portugaise pour en arriver à un repositionnement nécessaire de la critique. Pour reprendre l'expression d'un titre célèbre de Marc Fumaroli, Quand l'Europe parlait français (2001), le Théâtre français à l'étranger était un des principaux vecteurs de ce que Jean-Claude Yon nomme, en 2008, l'Histoire d'une suprématie culturelle ${ }^{3}$. Or cette suprématie implique l'exportation de certains modèles culturels et artistiques sous-jacents à une nouvelle vision de l'homme et de la société à laquelle il faut ajouter des conditions favorables à son épanouissement comme, par exemple, l'absence d'institutionnalisation des droits d'auteur. II faut dire aussi que «la France du XIXe siècle est le premier pays, en Europe et dans le monde ", comme l'affirme Jean-Claude Yon, "à se doter d'une véritable industrie théâtrale capable de produire en grande quantité des ouvrages destinés à un très large public » (Yon, $2008: 8)$.

Sous l'occupation napoléonienne, aussi bien à Lisbonne qu'à Rio de Janeiro, s'enracine un goût prononcé envers les ballets patriotiques qui étaient en vogue sous la France révolutionnaire. Véritable chronique des invasions, les sujets abordés retraçaient le triomphe des troupes anglo-portugaises et les batailles militaires. Comme l'avait déjà signalé Teófilo Braga, c'est sur les planches qui se jouaient les idéaux politiques. Bien que le théâtre, à l'époque, privé de sa nationalité et de la production de créations dramaturgiques nationales d'envergure, vive essentiellement de traductions et imitations du théâtre italien (surtout Métastase) et du théâtre français (Molière, Voltaire, Baculard d'Arnaud, Crébillon, entre autres), les manifestations théâtrales d'ordre politique occupaient un large pourcentage dans la vie de certaines salles de spectacle à Lisbonne. Les elogios dramáticos ${ }^{4}$ et les allégories dramatiques, devenus à la mode depuis des décennies, continuaient, plus que jamais, à attirer la foule au théâtre. Les occurrences artistiques là-dessus qui découlent des rapports entre la France et le Portugal au début du XIXe siècle sont nombreuses. Rappelons

\footnotetext{
${ }^{3}$ On l'a deviné, on reprend ici le titre et le sous-titre de l'ouvrage de Jean-Claude Yon publié, en 2008, aux éditions Nouveau Monde, à Paris.

${ }^{4}$ Teófilo Braga nous parle de ce phénomène artistique en ces termes : «O emprezario do Theatro da Rua dos Condes em 1809, Manoel Baptista de Paula, primava n'este género hybrido de espectáculos em que dispendeu milhões de cruzados para celebrar os annos de Dom João VI, de todos os príncipes, e alliados de Inglaterra » (Braga, 1871: 6).
} 
deux exemples. Tout d'abord, l'elogio dramático créé par Francisco Joaquim Bingre pour signaler la célébration du traité du 7 vendimiaire de l'an X (1801) entre Paris et Lisbonne. Ensuite, la comédie O Segundo Lusbel, Napoleão Bonaparte créée à l'occasion de la capitulation de Junot et de la signature de la Convention de Sintra (1808). Cette célébration fait l'objet d'une publication intitulée Desgraça de Bonaparte originada da liberdade, independencia e ventura de Hespanha e do abatimento da França pelos erros politicos d'aquelle tyrano qui circule pendant l'été 1808 et qui signale son extension dans le milieu théâtral :

No grande Theatro do mundo se representa a famosa Comedia de magica, intitulada O Segundo Lusbel, Napoleão Bonaparte, com muitas mudanças de Theatro, vistosissimos ornatos e tramoias nunca vistas. Faz o papel de Lusbel ou primeiro galan, o suave Napoleão; Godoi, de traidor, e terceiro galan; Dupont, de gracioso; Murat, de arlequim e tramoista; Talleyrand pinta os ornatos; e junto com Champagni e comparsa dos Senadores, move as machinas do theatro. Haverá além d'isto duas operasinhas traduzidas do italiano, cujas a primeira tem por título: A filha de Seipião, e a segunda a Sombra de Bruto. Seguir-se-há o entremez Foste buscar lã e vieste tosquiado, ou os ratos na ratoeira. Concluindo a funcção que se espera ser da acceitação de tão respeitável publico, com um fim de festa, em que a Andalucita a a Aragoneza bailarão o Sapateado com summa soltura sobre as tripas do gracioso: tudo novo e subido (Braga, 1871: 8-9).

José Arcúrsio da Neves, devenu premier historien des défaites des troupes napoléoniennes (Neves, 1810), allié à la profusion d'une littérature pamphlétaire qui qualifiait Napoléon de tyran des nations, peut bien avoir joué un rôle dans la transposition de cet esprit dans le milieu artistique et théâtral. Si au moment de l'entrée des troupes de Junot sur le territoire national on essaie de véhiculer, au départ, un climat de bonne entente introduite par la Gazeta de Lisboa, devenue le porte-parole de l'occupant français sous l'égide de l'Aigle impérial, très vite, des périodiques comme Leal Português et Minerva Lusitana deviennent le signe de la rébellion contre l'envahisseur. II faut dire que l'insurrection commence par des écrits élaborés en Espagne et que, dans les années qui suivent, des centaines de pamphlets sont édités par la Imprensa Régia et la Imprensa Real de l'université de Coimbra. Ces pamphlets restent, d'ailleurs, la plupart du temps, anonymes signés par un «portugais patriote » ou « un amant de la patrie » et louent, comme sur la scène théâtrale, la victoire de l'armée anglo-portugaise commandée par Wellington. 
Les manifestations artistiques étaient plus récurrentes, on le sait, au théâtre $S$. Carlos où défilaient les artistes très connus de la scène européenne, surtout d'origine italienne. Adrien Balbi, dans son Essai Statistique sur le royaume de Portugal e de l'Algarve (1822), avoue que le théâtre $S$. Carlos était dominé pendant la dernière décennie du XVIIle siècle par la présence italienne et que ce fut seulement après l'expulsion des troupes napoléoniennes que ce théâtre reçoit des artistes venus de l'opéra de Paris, de Bordeaux et de Lyon. On sait que les chorégraphes Lefebvre et Antoine Cairon sont venus à Lisbonne et ont monté, en 1814, le spectacle de Dauberval, La fille mal gardée, qui était un des modèles du genre en Europe.

Or, justement, pendant les invasions, les manifestations qu'on trouve dans ce domaine relèvent essentiellement de l'initiative de l'occupant étranger, français ou anglais, dans le seul espace théâtral prestigieux à ses yeux, le théâtre S. Carlos.

Tel est le cas du spectacle commandé par Junot en été 1808 dans ce théâtre pour fêter l'anniversaire de Napoléon. II s'agit d'un opéra du compositeur portugais Marcos Portugal intitulé Demofoonte. Des commandes du même genre ce sont multipliés de l'autre côté de la frontière et on peut se douter que d'autres initiatives semblables se soient perpétuées au moment des invasions sur le sol national. À titre d'exemple, P. Bohigas Tarragó nous raconte dans son ouvrage Las compañías dramáticas extranjeras en Barcelona (1946) comment les autorités françaises, au moment des invasions françaises en 1810 ont mis sur pied une saison théâtrale au théâtre de Santa Cruz en invitant une troupe française afin d'y représenter un opéra (La nuit champêtre de Saint-Aubin), une comédie (Shakespeare amoureux d'Alexandre Duval) et une tragédie burlesque (Fureurs de l'amour de Joseph Rochelle) qui a d'ailleurs tenu l'affiche pendant quelques mois. D'autre part, l'exemple de théâtre du Fondo à Naples qui accueille la troupe de Larive au mois de juillet 1807 sous l'emprise enthousiaste de Joseph Bonaparte est révélateur de ce point de vue.

La domination anglaise a touché aussi le seul espace théâtral véritablement digne de ce nom à Lisbonne. Le prestige atteint par le théâtre S. Carlos au cours des invasions françaises est redoublé par les résolutions de Beresford (loi du 3 février 1812) puisque cette salle de spectacle reçoit la compagnie du théâtre de la Rua dos Condes et garde le monopole de l'activité théâtrale à l'époque, sujette dorénavant, pour le plaisir des officiers anglais, à la représentation de pièces italiennes en musique5.

\footnotetext{
${ }^{5}$ Voici un extrait de ce décret daté du 3 février 1812 et signé par le gouverneur du royaume Alexandre José Ferreira Castelo : Havendo sua alteza real tomado em consideração, que a Sociedade do Theatro Nacional da Rua dos Condes, de que he director Manoel Baptista de Paula, recebendo hum moderado auxilio do Governo, tem sustentado este publico Espectaculo desde o seu estabelecimento em Dezembro de 1809, dando constantes provas do seu patriotismo, já na escolha de Peças próprias para o promover, já na applicação do producto das representações do primeiro domingo de cada mez para a caixa militar, e de outra a benéfico do cofre do resgate dos Cativos, cuja total importancia montou no anno próximo passado a perto de vinte mil cruzados; e já finalmente esmerando-se em mostrar o seu amor, respeito, e lealdade ao mesmo augusto Senhor, e sua real família, por meio de Espectaculos de grande apparato e despeza, com que tem festejado os faustos dias de seus
} 
Madame la duchesse d'Abrantes racontait d'ailleurs dans ces Mémoires deux épisodes de son contact avec la vie théâtrale lors de son séjour à Lisbonne avant les invasions, en 1805, lorsque son mari, le général Junot, est nommé ambassadeur à Lisbonne. Le tableau qu'elle dresse pour le théâtre $S$. Carlos et pour le théâtre du Salitre traduit bien toute la distance qui séparait l'art dramatique dans ces deux espaces de représentation théâtrale dans la capitale portugaise :

L'opéra de Lisbonne était à cette époque le plus fameux de l'Europe. La Catalani, alors dans son beau temps, était prima dona. Le soprano était Matucchi, venant après Crescentini, qui ne recommença pas l'année théâtrale, et partit pour Madrid après notre arrivée à Lisbonne. Le père noble était Monbelli, excellent acteur et bon ténor; puis Olivieri, bonne bassetaille. Voilà pour l'opéra séria. La Guaforini, Naldi, un ténor dont j'ai oublié le nom, voilà pour l'opéra buffa. Mettez ensuite dans cette liste les noms de Fioraventi, compositeur de l'opéra buffa, et Marcos Portogallo, compositeur pour l'opéra séria, en y ajoutant Caravita pour le libretto, et vous aurez l'idée de ce qu'était le théâtre de Lisbonne en 1805 et 1806. Quant au théâtre portugais qu'on appelle Teatro do Salitre, il était affreux. La salle était sombre et sale. Les acteurs détestables. Je fus voir un jour Gabrielle de Vergy, traduite en portugais, je commençais à comprendre un peu la langue, mais j'aurais autant compris du chinois que les AON des acteurs; ils avaient l'air de braire. Quant aux costumes, ils étaient à la grâce de Dieu, qui ordinairement ne s'en mêle pas. Lorsque Fayel arrive blessé dans la prison de Gabrielle, l'acteur voulant avoir l'air ensanglanté, à défaut d'un autre, s'était fait une immense tâche rouge sur un linge blanc... c'était hideux à voir. Tout à coup je m'aperçois que la blessure s'enlève... Et mon Dieu, dis-je à Junot, regardez donc, il me semble que la blessure de Fayel est à son menton... C'était vrai. Il était venu dans l'idée du Roscius lusitanien de coudre une grande loque de gaze

annos, assim como se tem empenhado em celebrar com iguaes demonstrações os dias natalícios dos soberanos da Gram Bretanha nossos íntimos alliados: Sendo de notória evidencia que a dita Sociedade não póde assim mesmo servir bem o publico sem que se transfira para hum local mais accommodado para os Espectaculos theatraes por sua extensão, e proporções, e sem que ajunte á representação das peças portuguezas a de algumas italianas em musica de maneira que os mesmos Empregados Britanicos, que presentemente se achão nesta capital, não fiquem privados de recreio que lhes póde afferecer o Theatro por ignorarem a língua do paiz. [...] He Servido approvar o estabelecimento de uma Sociedade composta de Actores e Artifices, que entrem com o seu trabalho, e de Accionistas particulares, que constituão hum fundo em Acções debaixo da Direcção do dito Manoel Baptista de Paula, e da immediata inspecção de Desembargador Sebastião José Xavier Botelho, auxiliando a mesma Sociedade com a mercê de oito casas de sortes, de que gozará até o carnaval do anno futuro de 1813, continuando-se-lhe depois este, ou qualquer outro socorro, que mais convier, se acaso o continuar a merecer: com obrigação de representar Dramas em linguagem, e Farças italiannas em musica. $\mathrm{E}$ sendo a Casa denominada de S. Carlos, além de sumptuosa, e propria de uma nação culta, a única em que se podem dar Espectaculos, que correspondão aos fins d'este estabelecimento, e por isso digna de conservar-se : He outro sim o mesmo augusto Senhor servido, que se fação as Representações na mencionada Casa, observando-se as Instrucções juntas assignadas pelo Desembargador do Paço, Alexandre José Freire Castello, Secretario do Governo na repartição dos Negócios do Reino e Fazenda, as quaes constituem o Regulamento Provisorio do Theatro Nacional, cuja boa organização, e melhoramento tanto podem concorrer para corrigir os vícios, adiantar a civilização, e inspirar as virtudes politicas, e sociaes, que fazem a felicidade dos imperios (Braga, 1871: 12-15). 
rouge après une autre loque blanche qui singeait l'appareil. Tout cela avait été mal attaché, et le crêpe rouge voltigeait au gré du vent de la coulisse, ce qui était fort pathétique. Les Portugais eux-mêmes n'allaient pas à leur théâtre national. Ils n'ont pas d'auteurs dramatiques. Les acteurs ne se forment pas, parce qu'il n'y a pas de public, et il n'y a pas de public, parce qu'il n'ya a pas de bons acteurs. Ce cercle vicieux existe pour beaucoup de choses, surtout au Portugal. (Abrantès, 1832 : 278280)

En tout cas, ce qui est sûr c'est que la fugue de la famille royale pour le Brésil à la fin de l'année 1807, les luttes militaires pour expulser l'envahisseur et les guerres libérales de 1820 jusqu'à 1836 contribuent à l'instabilité sociale politique et culturelle qui explique le ralentissement des programmes artistiques en salle de spectacle et le retard du pays en matière théâtrale. Mais le Portugal comme les autres pays du sud de l'Europe n'échappe pas, à l'aube du romantisme, aux vents rénovateurs qui soufflent de Paris. Les mutations politiques dans la Péninsule du début du siècle coïncident avec l'éclosion d'une nouvelle sensibilité dramatique et le besoin de rénovation de la scène nationale qui se trouve alors en pleine décadence.

Au Portugal, l'initiative aristocratique joue, tout d'abord, il faut bien le dire, un rôle très important au niveau du développement de l'art théâtral, notamment dans le domaine privé. Citons à titre d'exemple le théâtre das Laranjeiras construit en 1820 par le Comte de Farrobo dans sa propriété qui avait d'ailleurs fait l'objet du choix du général Junot pour s'installer dans la région. Ce théâtre accueille, la nuit du 4 décembre 1826, l'ambassadeur de France, le peintre Vandreil et Castellane, maréchal de Napoléon III venu à Lisbonne en mission militaire et diplomatique pour y assister à la représentation de la comédie L'amant et le mari de J.F. Roger et Jouy ainsi qu'à l'opéra Gli Aventuriere de Cordella et Giordani. Castellane, colonel de l'escorte militaire des derniers mois du royaume de Napoléon I ${ }^{\mathrm{e}}$, célèbre par ses campagnes en Espagne, en Allemagne et en Russie, registre ses impressions de cette nuitlà dans ses Mémoires et n'hésite pas à comparer ces moments culturels avec les fêtes les plus pompeuses des Tuileries à l'époque de l'impératrice Joséphine, femme de Napoléon ler.

Vingt ans après la première tentative napoléonienne d'introduire les artistes français sur la scène péninsulaire, les conditions étaient réunies pour que le public puisse enfin applaudir les grands succès parisiens dont la presse à vocation artistique s'employait d'ailleurs à faire la diffusion. L'Europe était prête à accueillir les troupes françaises en tournée et à édifier les nombreux «Théâtres-Français » destinés à un public d'élite qui maitrisait pleinement le français. On y assiste alors à l'un des phénomènes les plus emblématiques du XIXe siècle avec des Théâtres-Français dans plusieurs pays. Pour ne 
citer que les plus représentatifs, limitons-nous à évoquer ici ceux de Saint-Pétersbourg, Moscou, Varsovie, La Haye, Berlin, Vienne, Hambourg, Copenhague, Turin, Naples et Madrid. Les troupes françaises itinérantes qui y jouaient contribuaient surtout à accélérer la diffusion massive du répertoire parisien à l'étranger en offrant, par la même occasion, à une élite sociale et à un public cosmopolite, le plaisir de la langue et du jeu théâtral français. Au Portugal, il est vrai qu'il faut attendre une dizaine d'années après le départ des troupes napoléoniennes pour assister à une certaine effervescence dans le secteur de la collaboration entre impresarios et salles de spectacles à Paris et à Lisbonne, qui culminera d'ailleurs avec les tournées de la troupe de Saint-Eugène (1822) et celle d'Emile Doux (1835). La venue de cette dernière au théâtre de la Rua dos Condes, entre 1835 et 1837, constitue non seulement l'exemple le plus emblématique de la permanence d'une troupe française à l'étranger, mais représente aussi un impact extraordinaire sur le champ culturel national que la critique semble avoir minorisé jusqu'à maintenant mais qu'il faut réhabiliter. Cette troupe favorise par son répertoire l'introduction, sur la scène portugaise, des grandes nouveautés en matière théâtrale. Grâce à elle, le public mondain averti vénère les grands noms de la scène parisienne et assiste aux représentations données en version originale. Dans ce sens, on accueille très mal, du côté d'une certaine presse de l'époque, la submersion de la scène par cette présence étrangère ainsi que l'excès de traductions de pièces mises en scène sur le théâtre. II va sans dire que, dans ces conditions, cette domination ne se limite pas à la diffusion du répertoire parisien sur la scène portugaise. En effet, bien que celui-ci soit à l'origine, en version originale ou bien en traduction/adaptation, de plus de $80 \%$ des représentations sur la scène à Lisbonne, c'est surtout l'importation d'une pratique théâtrale et d'un certain savoir-faire qui aura un impact mesurable sur le monde théâtral portugais à l'aube du Romantisme. Si l'impact direct de la permanence de cette troupe française sur la scène théâtrale portugaise ne s'est pas révélé à la hauteur de l'édification du drame national, concourant avec les innombrables traductions d'un nouveau goût dramatique à l'heure de Paris, il reste néanmoins significatif du côté de l'histoire de la mise en scène et de la naissance de troupes nationales qui doivent beaucoup au répertoire français contemporain du théâtre du Gymnase Dramatique, du théâtre de la porte SaintMartin ou du théâtre des Variétés, entre autres. Ne l'oublions pas, la collaboration francoportugaise joue là aussi un rôle majeur puisque Almeida Garrett décide de charger les acteurs Paul et Manuel Baptista Lisboa de la classe de déclamation au Conservatoire qu'il vient de créer. Paul, mû par le défi de cette mission, déclare dans son exposition officielle du 27 décembre 1836 qu'il se propose de changer la situation afin d'améliorer l'art dramatique portugais et doter le pays d'un théâtre dont il pouvait être fier. Émile Doux, quant à lui, reste à Lisbonne pendant des décennies à la tête de la direction de différentes salles de spectacle dont le théâtre National jusqu'à 1840, le Salitre (1843-47), le Gymnase (1848), D. Fernando 
(1849) avant sont départ définitif au Brésil. Ses qualités de metteur en scène lui ont permis d'exercer non seulement une influence sur le renouveau de l'art dramatique et de la mise en scène au Portugal, mais aussi sur la formation une génération de comédiens dont Emilia das Neves, la plus grande tragédienne portugaise du XIXe siècle, reste l'emblème majeur. N'oublions pas, à ce propos, qu'Emile Doux fut responsable pour la mise en scène au théâtre de la Rua dos Condes, en 1838, du premier grand succès romantique sur la scène portugaise, l'Auto de Gil Vicente de Garrett dans lequel la belle Emilia a amorcé une carrière triomphale.

Le rôle social et civilisateur accordé au théâtre par le romantisme fit de cet art un véritable enjeu politique. La vie théâtrale prenait une place importante dans les préoccupations des lisboètes et une bonne trentaine de journaux lui furent consacrés à partir de la fin des années 1830. Cette presse, il faut bien le dire, ne ménagea pas Emile Doux. À son style français développé au théâtre de la Rua dos Condes on voulait opposer un style national naissant créé par une jeune troupe dirigée par l'acteur Fructuoso Dias au théâtre du Salitre ${ }^{6}$. Le public, lui, préférait le Condes - appelé le Théâtre Français - et la bonne société de la capitale aimait à s'y montrer.

En outre, cette opposition ne favorisait pas l'attribution des subventions par l'Etat, comme l'observe si bien Alexandre Herculano dans une lettre adressée à Almeida Garrett en 1839 :

Admitamos, caso negado, que cumpria agora haver um concurso para o governo pagar este dinheiro a seus donos ; seria exequível esse concurso? A carta de V. Ex e a nossa própria convicção nos diz que não. Nenhum dos dois diretores pode satisfazer as condições principais e necessárias: nenhuma das companhias é completa: para os caracteres cómicos incontestavelmente tem o Salitre melhores atores; para os caracteres médios e trágicos tê-los-á melhores a Rua dos Condes: 0 Salitre poderá vir a ter mais abundância de dramas originais; a Rua dos Condes talvez mais apropriado vestuário e melhor cenário. De que lado estará a vantagem? Não o decidiremos nós, mas o que é indubitável é que nenhum deles poderá preencher sequer as condições capitais. Com o tempo talvez ambos as possam aceitar e cumprir, mas já, é impossível (Herculano, s.d.: 16-17).

Dans ces conditions, une seule issue était possible afin de subvenir au manque de financement: la diversification du spectacle afin d'attirer un public plus vaste et plus diversifié du point de vue social qui n'était pas absorbé, bien sûr, par le théâtre $S$. Carlos.

\footnotetext{
${ }^{6}$ Cette opposition est très nette entre deux journaux de l'époque (1838), Atalaia Nacional dos Theatros et le Desenjoativo theatral, partisans des deux théâtres et des deux écoles dramatiques.
} 
Une soirée théâtrale au théâtre du Salitre surtout était composée de plusieurs pièces où étaient intercalées des pièces de musique, quelques danses et des spectacles de magie et d'acrobatie.

Une réforme du théâtre et l'édification d'un théâtre national était une nécessité impérieuse ressentie par certains intellectuels dès lors. La situation politique ne permettait pas son éclosion et ce projet ne sera reformulé que le 28 septembre 1836 lorsque le ministre Manuel da Silva Passos signe un arrêt chargeant Almeida Garrett de proposer un plan pour la fondation et l'organisation d'un théâtre national susceptible de contribuer à la civilisation et à l'instruction morale de la nation. Un mois et demi après Garrett s'exécute et dès le 15 novembre un nouveau décret officialise ce projet. Celui-ci conçoit le théâtre dans une globalité jamais égalée au Portugal : il souligne la nécessité de former de nouveaux acteurs, de créer un répertoire national et de construire un théâtre. Le 22 novembre suivant est créée I'Inspection Générale des Théâtres, dirigée par Garrett. On voit avec quelle rapidité les évènements se succèdent ce qui dénote effectivement une volonté politique d'attribution d'une place au théâtre dans son rôle formateur des mentalités, non pas uniquement auprès du public, bien sûr, mais aussi auprès de ceux que en sont les artisans, les auteurs et les acteurs. II est vrai qu'on espère ainsi que les uns prennent conscience et contribuent à l'émergence d'une dramaturgie aux couleurs locales nationales et que les autres travaillent en vue de leur formation et de leur performance sur scène. Mais il est vrai aussi que la présence massive de l'invasion française en matière théâtrale, matérialisée par la dispute entre les troupes françaises et quelques troupes nationales en vue d'un renouvellement persistant du répertoire des salles dans la capitale portugaise, constitue un facteur essentiel vers un repositionnement du regard qu'il faut désormais projeter sur l'évolution de l'histoire culturelle au Portugal à cette époque face à l'hégémonie française. 


\section{Bibliographie}

ABRANTES, Laure Junot (1832). Mémoires de $M^{\text {me }}$ la Duchesse d'Abrantès, ou souvenirs historiques sur Napoléon, la Révolution, le Directoire, le Consulat, l'Empire et la Restauration. Tome septième. Paris : Ladvocat Librairie de S.A.R. le Duc d'Orléans. BALBI, Adrien (1822). Essai Statistique sur le royaume de Portugal et de l'Algarve comparé aux autres États de l'Europe et suivi d'un coup d'œil sur l'état actuel des Sciences, des lettres et des Beaux-Arts parmi les Portugais des deux hémisphères. Paris : Rey et Gravier, 2 vol.

BRAGA, Teófilo (1871). História do teatro português. Garrett e os dramas românticos. Porto: Imprensa Portuguesa editora.

BOHIGAS TARRAGÓ, P. (1946). Las compañías dramáticas extranjeras en Barcelona.

Barcelona: Investigaciones, nำ 2 .

FUMAROLI, Marc (2001). Quand l'Europe parlait français. Paris: Fallois.

Herculano, Alexandre (s.d.). Cartas, II. Amadora: Bertrand.

IAN/TT. (1794). Intendência Geral de Polícia: Contas para as Secretarias. Liv. IV, 5 de Novembro, fl. 212 v.

MARTINS, Wilson (1978). História da inteligência brasileira. $2^{\mathrm{a}}$. ed. São Paulo: Cultrix/Editora da Universidade de São Paulo. vol. 2.

NEVES, José Acúrsio das (1810). História geral da invasão dos franceses em Portugal e da restauração do Reino. $1^{\circ}$ tomo. Lisboa: Oficina Simão Tadeu Ferreira.

RUDERS, Carl Israel (2002). Viagem em Portugal, 1798-1802. Tradução de António Feijó, prefácio e notas de Castelo Branco Chaves. Lisboa: Biblioteca Nacional, vol.1. VICENTE, António Pedro (1999). «Panfletos anti-napoleónicos durante a guerra peninsular. Actividade editorial da real Imprensa da Universidade», Revista de História das Ideias, vol. 20, pp. 101-130.

YON, Jean-Claude (dir). (2008). Le théâtre français à l'étranger au XIXe siècle. Histoire d'une suprématie culturelle. Paris : Nouveau Monde éditions. 